Editorial

\title{
Driving Skills in Older Adults
}

\author{
Michael Falkenstein *
}

Institute for Work, Learning and Aging (ALA) Hiltroper Landwehr 136, 44805 Bochum, Germany; E-Mail: falkenstein@ala-institut.de

* Correspondence: Michael Falkenstein; E-Mail: falkenstein@ala-institut.de

Academic Editor: Michael Falkenstein

Special Issue: Driving Skills in Older Adults

\section{OBM Geriatrics}

2020, volume 4, issue 3

doi:10.21926/obm.geriatr.2003130
Received: August 11, 2020

Accepted: August 11, 2020

Published: August 19, 2020

The percentage of the older population is rapidly increasing worldwide. It has been estimated that by the year 2050, one-third of the population in the OECD countries will be above 65 years. This demographic change will increase the number of older people who want to or have to stay mobile because of economic conditions. Mobility is a key to healthy aging as it is related to the basic human need for physical movement. Mobility is associated with a person's physical and psychological well being. Driving one's car is a popular mode of transport in developed countries. Cessation of driving can lead to a range of detrimental consequences, such as a decrease in social activities, which impacts cognitive health. Besides, restriction to car driving can force older people to use less safe modes of transport, making them more vulnerable to accidents. Hence, older adults should continue driving their cars as much as possible.

Driving is an activity that takes place in a dynamic environment requiring sensory, motor, and cognitive functions. With increasing age, a decline in most of these functions has been observed. However, changes in functions differ among individuals and are influenced by a multitude of environmental and lifestyle factors. Such functional changes may affect the performance of

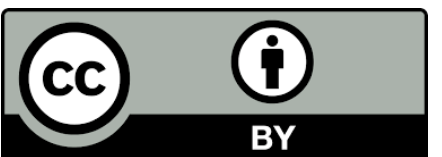

(C) 2020 by the author. This is an open access article distributed under the conditions of the Creative Commons by Attribution License, which permits unrestricted use, distribution, and reproduction in any medium or format, provided the original work is correctly cited. 
everyday tasks, especially complex activities. Indeed, visual and cognitive factors can explain most of the age-related changes in the ability to drive safely.

Since most of the traffic-relevant information is perceived by the visual system, age-related changes in vision are the most relevant for driving. Such changes include reductions in visual acuity and contrast sensitivity, increased glare sensitivity, and reduced field of view. Motor changes include diminishing muscle strength and movement speed, which may slow down emergency actions or prolong braking time. Furthermore, neck flexibility is essential during driving and is strongly reduced with age. Finally, motor coordination and dexterity also deteriorate with increasing age.

Cognitive issues in older adults are often overlooked despite their high importance in driving. In particular, the so-called executive functions, which control lower-level functions, are highly relevant for driving. Executive functions include inhibition of irrelevant information, updating of memory, and switching between tasks. Besides, they control attention, which is necessary for visual search and attention switching and distribution. For example, visual search, that is, the scanning of the visual scene to detect a target stimulus is mostly impaired in people in their 60s. Hence, in real traffic, important targets and threats are possibly detected later or none by older adults.

Cognitive problems increase in mild cognitive impairment $(\mathrm{MCl})$, which is present in many older adults. Drivers with $\mathrm{MCl}$ have enhanced driving problems than those without $\mathrm{MCl}$.

Safe driving depends not only on skills but also on personality factors like self-rating, confidence, risk acceptance, or sensation-seeking. Most of the older adults are overconfident regarding their driving skills and rate themselves as good or excellent drivers regardless of their actual performance. This may impair their motivation to take measures to improve their driving.

Despite impaired functions and overestimated driving performance, older adults often drive inconspicuously. This is because routine traffic situations such as highway driving rely mainly on highly automated processes, which show a less age-related decline. Moreover, to cope with more complex situations, many older adults develop compensation strategies such as slow driving and selection of well-known routes. Also, compensation mechanisms such as a stronger preparation in complex situations are frequently observed among the older population. However, some of those strategies are not satisfactory since they may encumber other drivers (e.g., slow driving) or the driver himself (e.g., avoidance of certain routes).

Nevertheless, the accident rate among older adults, based on distance driven estimates, is relatively high. In particular, drivers aged above 75 years who drive less than $3000 \mathrm{~km}$ per year have the highest accident risk. Moreover, $75 \%$ of drivers aged 75 and above who are involved in an accident are primarily responsible for the accident. A closer look at the accidents shows that they occur in specific situations such as turning, driving backward, and complex traffic junctions. However, accident rates underestimate the problems since, in most critical situations, accidents can be avoided by the driver himself or by other traffic participants. More often, near-accidents likely happen that do not show up in the statistics but may be remarked by fellow passengers.

Apart from having problems in certain situations, older adults are also more vulnerable than younger adults due to their fragile body structure. For example, the bones and ribs are not flexible in old age, which can be aggravated by osteoporosis, a common disease in older adults. Such lower flexibility usually leads to more serious injuries, and even safety belts could cause fatal injuries in old adults. 
Even more vulnerable are older adults who drive bicycles. Riding a bicycle not only requires the skills of car driving, that is, coping with complex situations and interacting with other drivers, but also the skills of handling the bicycle, which is not performed automatically as handling a car. Many older adults who have not used their bicycles for years have poor cycling skills, which are often not realized by the cyclist. Hence, accident rates among older cyclists increase steadily and mainly while using e-bikes with their weight and speed.

Another issue among older adults is fatigue. Many accidents happen when the driver is fatigued. Most of the older adults suffer sleeping problems and hence are more fatigued than younger adults. On the other hand, there is some evidence that older adults are more resistant to fatigue. The question is about the marginal conditions under which older drivers are more or less vulnerable to fatigue than younger drivers.

These facts show that measures are necessary to increase safety and support for older drivers. In several countries, to increase safety, medical and psychological tests are mandatory for older adults to renew their driving license. However, this does not necessarily mean that safety is increased. For example, the number of fatal accidents in Finland, which requires a medical test for older drivers, is not lower than in Sweden, a country with no such test. One reason for such failures is the type of tests required. Usually, only cursory and short medical tests are used. When cognitive tests are given, they are often designed for dementia diagnosis, such as the Mini-Mental State Examination (MMSE). Such tests are unsuitable for measuring the physical, sensory, and cognitive skills that are essential for driving.

The most important issue is the identification of unfit older drivers. An obvious and direct method is an on-road driving assessment, usually conducted by a driving instructor, who is sometimes accompanied by a traffic psychologist. Meanwhile, there are standardized and even PC-based driving protocols that aim at more objectivity. However, such on-road assessments are costly and require appropriate equipment and time resources. Besides, the real traffic is not always challenging, which depends on the time and location of the assessment ride, and of course, on the competence and experience of the driving instructor. Hence, the elderly may show no problems even though they would probably be revealed in complex situations. An alternative is to drive using a driving simulator, which has the advantage of administering and repeating sufficiently difficult scenarios. As with driving tests in real traffic, this methodology requires adequate equipment and skilled experts to yield reliable results. Also, the feeling of being in real traffic is absent in simulators, while there is often simulator sickness with older adults. $A$ third and promising alternative is to administer off-road tests for cognitive function as well as visual and motor functions that are important for driving. This reminds of the periodic car inspection in which functions of the car and not its behavior in real traffic is checked. The crucial issue with such testing is the selection of the most appropriate tests for sufficiently predicting driving fitness and accident rate. Single and ill-chosen tests have no predictive power. However, carefully compiled test batteries that include tests of the most important functions relevant for driving appear to have high predictive power. Such off-road assessments should also include interviews that ask for risk factors such as avoidance behavior, reports of unsafe driving by relatives, the number of (minor) accidents in the past years, and reduced driving practice.

All those assessment methods are only meaningful if they are accompanied and followed by measures to support older drivers, and in particular, those who have driving problems. Otherwise, assessments are likely avoided if voluntary, and dreaded if mandatory. Such measures could either 
be information and guidance campaigns, the design of age-friendly traffic and car environments, or training measures that are aimed at improving the individual driving skill of an older driver.

Information campaigns address the older public, giving information about factors that influence driving fitness (e.g., certain diseases and drugs), about strategies on how to cope with certain driving situations, and about training possibilities to increase driving performance. They are relatively easy to organize. However, the success of such campaigns depends on whether they are accompanied by practical courses in groups.

Certain traffic situations, such as turning left at complex crossroads, are particularly difficult for older drivers and can be decreased by age-friendly street design. For example, left turns should be protected by traffic lights or by well-visible guidelines. Also, at crossroads or roundabouts, distracting and traffic-irrelevant information such as advertisements should be minimized. Complex areas should be structured and traffic routing distinctly marked by coloring. On the other hand, well-designed car technology such as high doors and seats and broad circumferential visibility can help elderly drivers. Above all, route guidance systems are highly important for the elderly since they reduce the need for memory and visual search. However, to be helpful and not distracting, such systems have to meet certain requirements.

The third measure is individual training for older drivers. The most straightforward training is to take driving lessons with a driving instructor in real traffic. They require well-trained driving instructors who know the problems of older drivers as well as sufficiently difficult traffic locations. If the training is properly conducted, such driving lessons can strongly improve driving fitness in older drivers, and also for poor drivers.

An off-road variant is the training of specific skills (such as visual search) or coping with difficult driving scenarios with the help of programs running on a personal computer or a driving simulator. The personal computer has the advantage of being affordable and widely available, so it might be possible to conduct such training at home, after proper instruction.

A further possibility is the direct training of functions that are necessary for driving, in particular motor, and cognitive functions. For example, head movements and strength can be trained and result in improvements in the trained functions.

In the cognitive domain, visuospatial skills and spatial attention can be trained; the latter results in better and longer driving skills. Besides, physical training is known to improve motor and cognitive functions. However, there are only a few studies which show improvements in driving fitness after undergoing such training. Since training can be conducted at home, further studies on this topic are warranted.

The present special issue aims at addressing most of the mentioned issues and any other issue concerning older drivers and how to help them keep driving as long as possible.

\section{Author Contributions}

Michael Falkenstein did all the research work for this study.

\section{Competing Interests}

The author has declared that no competing interests exist. 


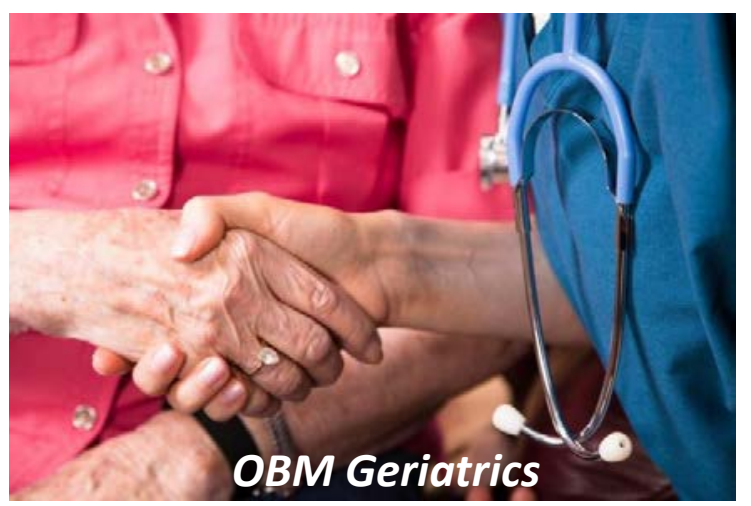

Enjoy OBM Geriatrics by:

1. Submitting a manuscript

2. Joining in volunteer reviewer bank

3. Joining Editorial Board

4. Guest editing a special issue

For more details, please visit:

http://www.lidsen.com/journals/geriatrics 